\title{
ESA'S SPACE-BASED DOPPLER WIND LIDAR MISSION AEOLUS - FIRST WIND AND AEROSOL PRODUCT ASSESSMENT RESULTS
}

\author{
A.G. Straume ${ }^{1}$, M. Rennie ${ }^{3}$, L Isaksen ${ }^{3}$, J. de Kloe ${ }^{4}$, G.-J. Marseille ${ }^{4}$, A. Stoffelen ${ }^{4}$, T. Flament ${ }^{5}$, H. \\ Stieglitz ${ }^{5}$ A. Dabas ${ }^{5}$, D. Huber ${ }^{6}$, O. Reitebuch ${ }^{7}$, C. Lemmerz ${ }^{7}$, O. Lux ${ }^{7}$, U. Marksteiner ${ }^{7}$, F. Weiler ${ }^{7}$, \\ B. Witschas ${ }^{7}$, M. Meringer ${ }^{8}$, K. Schmidt ${ }^{8}$, I. Nikolaus ${ }^{9}$, A. Geiss ${ }^{10}$, P. Flamant ${ }^{11}$, T. Kanitz ${ }^{1}$, D. \\ Wernham $^{1}$, J. von Bismarck ${ }^{2}$, S. Bley ${ }^{2}$, T. Fehr ${ }^{1}$, R. Floberghagen ${ }^{2}$, T. Parinello ${ }^{2}$ \\ ${ }^{1}$ European Space Agency (ESA) ESTEC, Noordwijk, Netherlands \\ ${ }^{2}$ European Space Agency (ESA) ESRIN, Frascati, Italy \\ ${ }^{3}$ European Centre for Medium-Range Weather Forecasts (ECMWF), Reading, United Kingdom \\ ${ }^{4}$ Royal Dutch Meteorological Institute (KNMI), de Bilt, Netherlands \\ ${ }^{5}$ MétéoFrance, Toulouse, France \\ ${ }^{6}$ DoRIT, Oberpfaffenhofen, Germany \\ ${ }^{7}$ DLR, Institute of Atmospheric Physics, Oberpfaffenhofen, Germany \\ ${ }^{8}$ DLR, Remote Sensing Technology Institute, Oberpfaffenhofen, Germany \\ ${ }^{9}$ University of Applied Sciences, Munich, Germany \\ ${ }^{10}$ Ludwig-Maximillians-University, Meteorological Institute, Munich, Germany \\ ${ }^{11}$ LATMOS, Institut Pierre Simon Laplace (IPSL), Paris, France \\ *Email: Anne.Straume@esa.int
}

\begin{abstract}
The European Space Agency (ESA) wind mission, Aeolus, hosts the first space-based Doppler Wind Lidar (DWL) world-wide. The primary mission objective is to demonstrate the DWL technique for measuring wind profiles from space, intended for assimilation in Numerical Weather Prediction (NWP) models. The wind observations will also be used to advance atmospheric dynamics research and for evaluation of climate models. Mission spinoff products are profiles of cloud and aerosol optical properties. Aeolus was launched on 22 August 2018, and the Atmospheric LAser Doppler INstrument (Aladin) instrument switch-on was completed with first high energy output in wind mode on 4 September 2018 [1], [2]. The on-ground data processing facility worked excellent, allowing L2 product output in near-real-time from the start of the mission. First results from the wind profile product (L2B) assessment show that the winds are of very high quality, with random errors in the free Troposphere within (cloud/aerosol backscatter winds: $2.1 \mathrm{~m} / \mathrm{s}$ ) and larger (molecular backscatter winds: $4.3 \mathrm{~m} / \mathrm{s})$ than the requirements $(2.5 \mathrm{~m} / \mathrm{s})$, but still allowing significant positive impact in first preliminary NWP impact experiments. The higher than expected random errors at the time of writing are amongst others due to a lower instrument outand input photon budget than designed. The instrument calibration is working well, and some of
\end{abstract}

the data processing steps are currently being refined to allow to fully correct instrument alignment related drifts and elevated detector dark currents causing biases in the first data product version. The optical properties spin-off product (L2A) is being compared e.g. to NWP model clouds, air quality model forecasts, and collocated ground-based observations. Features including optically thick and thin particle and hydrometeor layers are clearly identified and are being validated.

\section{INTRODUCTION}

The European Space Agency (ESA)'s Living Planet Programme includes two types of complementary user driven missions: the research oriented Earth Explorer missions and the operational service oriented Earth Watch missions. The Earth Explorer missions are proposed, assessed and recommended by the European Scientific community, and address the scientific challenges set out in the ESA Living Planet Programme and the ESA Earth Observation Science Strategy [7]. Following the recommendations at the ESA User Consultation Workshop in Granada, Spain in 1999, ESA implemented Aeolus as an Earth Explorer Core mission. The Aeolus mission industrial prime is Airbus Defense and Space (ADS) United Kingdom, and the Aladin instrument prime is ADS France. The Aladin instrument lasers are built by Leonardo, Pomezia, Italy. 


\section{THE AEOLUS MISSION}

\subsection{Mission motivation and objectives}

The Aeolus mission selection was motivated by the need for more abundant direct wind profile measurements in the World Meteorological Organization (WMO) Global Observing System (GOS), which is used as input to weather forecast models world-wide [3]. Currently, the global distribution of conventional wind profile measurements (e.g. radiosondes, aircraft winds, ground-based wind lidars and radars) is not homogenous and mainly located over land in the Northern Hemisphere. Winds are also inferred from temperature soundings for the large-scale wind fields in the extra-tropics. Scatterometers provide surface winds over the oceans (single level), and Atmospheric Motion Vector (AMV) winds deduced from cloud, aerosol, humidity or ozone tracking are limited in vertical coverage and have less accurate height-assignments. The lack of 3D homogenous wind field sampling, in particular in the tropics, over the oceans and in polar areas, hence leads to difficulties in constraining atmospheric models and limits the studying of key processes in coupled climate systems. Aeolus winds will contribute to mitigate the current wind observation deficit.

The primary mission objective is to demonstrate the DWL technique for measuring wind profiles from space, intended for Near-Real-Time (NRT) assimilation in NWP models. Meteorological Centers world-wide are currently preparing to ingest Aeolus winds near-real-time in their weather models as soon as the data quality is of sufficiently good quality. Aeolus is expected to allow for significant forecast improvements. The wind observations will also be used to advance atmospheric dynamics research and for evaluation of climate models.

\subsection{Instrument concept}

Aeolus embarks a single payload, the direct detection Doppler Wind Lidar instrument Aladin. It is a pulsed UV lidar (355 nm, circularly polarized, $50 \mathrm{~Hz}$ ), measuring continuously along the orbit (Figure 1, left panel). Its high spectral resolution concept allows for the detection of the parallel polarized molecular (Rayleigh) and particle (Mie) backscattered signals from the circularly polarized emitted light in two separate channels, each sampling the backscatter in 24 vertical height bins (Figure 1, right panel). This allows the instrument to measure winds both in clear and (partly) cloudy conditions down to optically thick clouds. The height of the wind measurement is detected by time gating. A quasiglobal coverage is achieved daily ( $\sim 16$ orbits per day) and the orbit repeat cycle is 7 days (111 orbits). The orbit is sun-synchronous with a local equatorial crossing-time of $6 \mathrm{am} / \mathrm{pm}$. A more detailed description of the instrument design and operation can be found in [3] and [6].
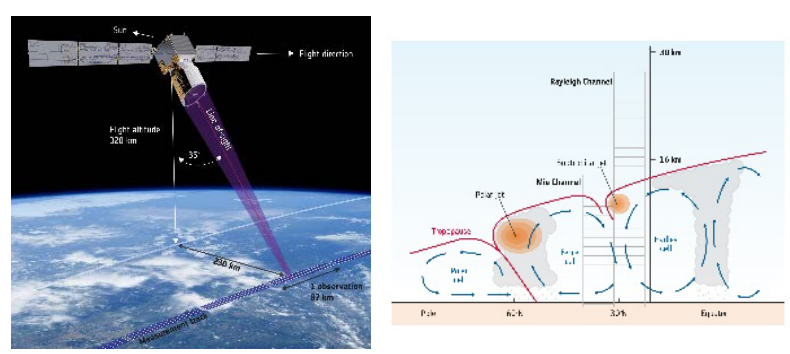

Figure 1: The Aeolus orbit, pointing and sampling characteristics (left) and an example of the vertical measurement binning by the two channels measuring winds from particle (Mie) and molecular (Rayleigh) returns (right).

\subsection{Products and product requirements}

The main product from Aeolus is horizontally projected line-of-sight (HLOS) wind profile observations (approximately zonally oriented) from the surface up to $25-30 \mathrm{~km}$ altitude (L2B product) [4]. The vertical sampling and range is configurable, and is being optimized in the operational phase of the mission. The atmospheric backscattered signal for the individual laser pulses are averaged on-board to yield $\sim 3 \mathrm{~km}$ measurements along-track. These measurements are further averaged on-ground to observations, representing horizontal scales up to $\sim 88 \mathrm{~km}$. The vertical resolution of the winds varies from 0.25 to $2 \mathrm{~km}$, adapted as a function of the underlying topography and/or climate zone. The HLOS wind observation random error (precision) requirement is $1 \mathrm{~m} / \mathrm{s}$ in the Planetary Boundary Layer (PBL), $2.5 \mathrm{~m} / \mathrm{s}$ in the free troposphere, and $3-5 \mathrm{~m} / \mathrm{s}$ in the stratosphere, and the bias (systematic error) requirement is $0.7 \mathrm{~m} / \mathrm{s}$. The wind profiles are classified into cloudy and cloud-free observations.

The Aeolus Level 2A product contains profiles of particle and molecular parallel polarized 
backscatter and extinction coefficients, scattering ratios, and backscatter-to-extinction ratios [5]. From these parameters it is possible to derive particle layer height, multi-layer cloud/aerosol stratification, cloud/aerosol optical depths, and some information on cloud/aerosol type.

Other products will be developed during the mission operational phase. ESA issues invitations to tender (ITTs) calling for further mission spin-off products and scientific applications (http://emits.sso.esa.int/).

\section{RESULTS}

First results of the Aeolus instrument characterization and calibration are presented in [1] and [2]. This paper shows first results of the Aeolus Level 2 product assessment.

\subsection{First results of the Aeolus L2 wind product assessment}

The initial assessment of the Aeolus primary product, the L2B wind profile observations, has been done by ECMWF and KNMI under ESA contract. ECMWF, KNMI and MétéoFrance have developed the Aeolus L2B processor and processing facility, including product quality monitoring using the ECMWF weather model. ECMWF hosts the Aeolus L2B processing facility, is currently performing NWP impact experiments and will actively assimilate the L2B product as soon as the quality is adequate. KNMI maintains and develops the L2B processor, with a special emphasize on the scene classification. Results from the first data quality assessment is shown in Figure 2, where Aeolus winds from one orbit are compared with ECMWF model winds projected onto the Aeolus line-of-sight.
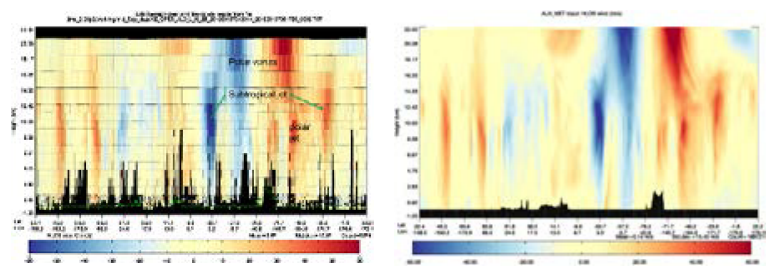

Figure 2: Aeolus L2B product winds combining observations from the Mie and Rayleigh channels over one orbit (September 15 2018), and collocated ECMWF model winds along the same orbit (M. Rennie).

The plots show a very good correlation between the large-scale wind features in the Aeolus observations and ECMWF model winds, such as sub-tropical jets and polar stratospheric jets. In Figure 3, Aeolus observation minus model background wind (o-b) statistics from 7 orbits are shown.

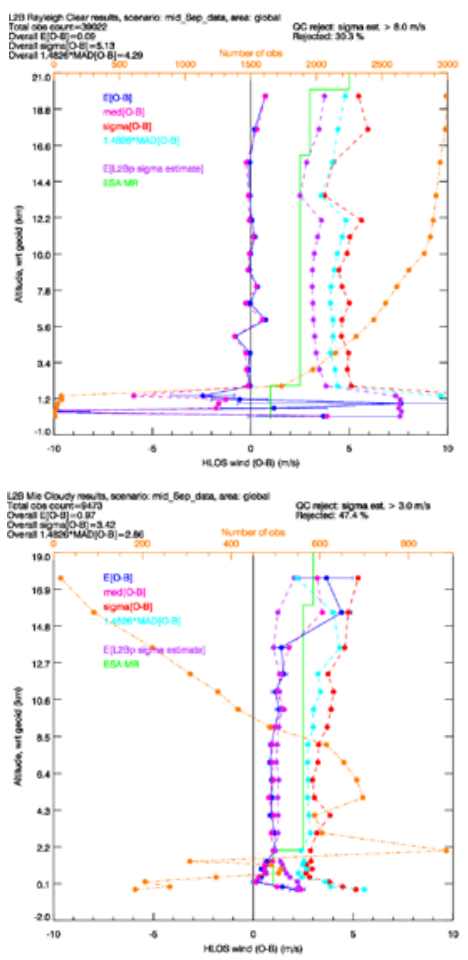

Figure 3: Aeolus Rayleigh (molecular return, top panel) and Mie (particle return, lower panel) wind observation monitoring statistics, 15 September 2018 (M. Rennie). Green: observation random error requirement, Blue: mean (observation (o) - ECMWF model background (b)), Red: $\sigma(\mathrm{o}-\mathrm{b})$, Pink: median value $(0-b)$, Magenta: scaled medium absolute deviation $(o-b)$, Purple: L2B processor estimated random error.

The Rayleigh wind (from molecular backscatter) deviation from the model background (Fig. 3 top panel, expressed as a robust estimate of the Aeolus observation standard deviation) is $4.3 \mathrm{~m} / \mathrm{s}$ between 2 and $16 \mathrm{~km}$ for this dataset, which is larger than the mission random error requirement $(2.5 \mathrm{~m} / \mathrm{s}$, [6]). The mean bias is $0.1 \mathrm{~m} / \mathrm{s}$ which is lower than the mission bias requirement $(0.7 \mathrm{~m} / \mathrm{s}[6])$. In the PBL (defined as $0-2 \mathrm{~km}$ altitude), the random errors are larger than the requirement, largely due to high-resolution vertical sampling leading to too low signal per height bin. PBL vertical sampling was intentionally optimized for ground detection investigations in this dataset. After the initial instrument calibration activities (first $\sim 5$ months), 
the vertical binning was changed to optimize the wind quality in the PBL. The Mie wind (from particle and hydrometeor backscatter) deviation from the model background (Fig. 3 lower panel, expressed as a robust estimate of the observation standard deviation) are $2.1 \mathrm{~m} / \mathrm{s}$ in the free troposphere and hence meet the mission requirements. The mean bias is $1 \mathrm{~m} / \mathrm{s}$ and higher than the mission requirements, because the bias correction using the instrument calibration measurements in the data processing was disabled. This was because the instrument characterization and calibration needed to be completed before the calibration processing could be switched on. With the upcoming processor deliveries and data reprocessing, it is expected that the product biases will be compliant with the requirements. First NWP impact experiments by $M$. Rennie at ECMWF show positive impact of the Aeolus observations in the ECMWF model tropical troposphere and southern hemisphere wind, humidity and temperature forecast at short-range relative to other observation types, which looks very promising. Further results from the Aeolus NWP impact assessment is expected to be available for the $29^{\text {th }}$ ILRC conference.

\subsection{First results from the Aerosol L2 optical properties products assessment}

The initial assessment of the Aeolus L2A backscatter and extinction coefficient profile observations, has been done by MétéoFrance under ESA contract. MétéoFrance has developed the Aeolus Calibration Suite (for L2B RayleighBrillouin scattering correction and L2A specific calibration) and the L2A atmospheric optical properties processors. An example of Aeolus L2A data from one orbit is shown in Figure 4, where cloud features up to $14 \mathrm{~km}$ in the tropics are visible. White areas indicate total signal attenuation. The assessments of the data quality are on-going at MétéoFrance, comparing the products to ground and space-based Atmospheric Optical Densities (AODs), ECMWF model clouds and CAMS air quality model aerosol analysis fields. Further results will be shown in the $29^{\text {th }}$ ILRC presentation.

\section{CONCLUSIONS}

First results from the Aeolus L2 wind and atmospheric optical properties product assessment show that the Aeolus Doppler wind lidar measurement principle has been proven from space. The Aeolus wind product quality is close to the mission requirements, and the activation of the instrument calibration processing is expected ensure the winds to meet the bias requirements. The Aeolus product quality is assessed by scientific teams world-wide with collocated ground-based, airborne and space-based measurements, weather and air quality models. Results from the Aeolus CAL/VAL and Science workshop in March 2019 will be shown at the $29^{\text {th }}$ ILRC.

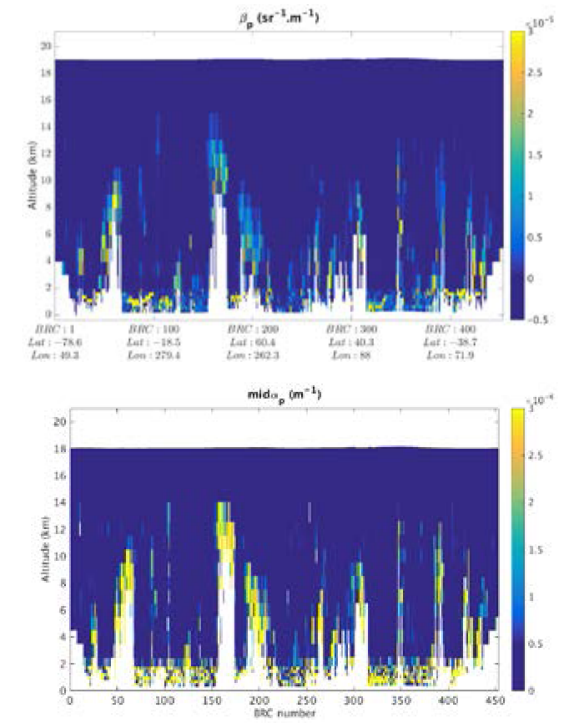

Figure 4: Aeolus L2A product particle backscatter (top panel) and particle extinction (lower panel) coefficient profiles for one orbit, 2 January 2019 (T. Flament, H. Stieglitz).

\section{REFERENCES}

[1] T. Kanitz et al., ESA'S Lidar Missions Aeolus and EarthCARE, Proceedings of the $29^{\text {th }}$ ILRC, this issue.

[2] O. Reitebuch et al., Initial assessment of the performance of the first wind lidar in space on Aeolus, Proceedings of the $29^{\text {th }}$ ILRC, this issue.

[3] ESA, 2008, ADM-Aeolus Science Report. ESA SP1311, 121 pp., available on http://www.esa.int/aeolus [4] D. Tan et al., 2017, Aeolus Level-2b Algorithm Theoretical Basis Document, available on https://earth.esa.int/aos/AeolusCalVal

[5] P. Flamant et al., 2017, ADM-Aeolus L2A Algorithm Theoretical Baseline Document, available on https://earth.esa.int/aos/AeolusCalVal

[6] ESA, 2016, ADM-Aeolus Mission Requirements Document, available on http://www.esa.int/aeolus [7] http://www.esa.int/Our_Activities/Observing_the Earth/The_Living_Planet_Programme 